\title{
Cooperative effects and slow dynamics of fluorescence intensity from quantum emitters in a dielectric
}

\author{
N.A. Lozing ${ }^{1,2, *}$, and M.G. Gladush ${ }^{1,2,3}$ \\ ${ }^{1}$ Institute for Spectroscopy RAS, 108840 Moscow, Troitsk, Russia \\ ${ }^{2}$ Moscow Institute of Physics and Technology, 141700 Dolgoprudny, Russia \\ ${ }^{3}$ Moscow State Pedagogical University, 119991 Moscow, Russia
}

\begin{abstract}
We study theoretically the possibility of spontaneous switching between dim and bright fluorescence modes from a cooperative ensemble of two-level atoms driven by a cw-laser. A numerical analysis of transient regimes and transformations of the fluorescence spectrum are reported.
\end{abstract}

We study a bistability of two-level light emitters that are collected in a cooperative ensemble. It is known from the theories and experiments that many-particle systems may exhibit collective behavior because of interactions, like dipole-dipole, that are allowed or enhanced under certain conditions. Emission of light from such ensembles may be different than that from an uncorrelated group. Coherent spontaneous emission, superradiance, collective fluorescence and accompanying effects have been studied for several decades. At the same time a great number of problems specific for many-particle systems remain a challenge for theorists. The problem we apply to in this work is the fact that any cooperative system is likely to become optically bistable [1,2]. In other words, there may be conditions for multiple steady-state populations and polarizations of the particles, realizable for the same external parameters. The presence of the material that hosts the light emitters brings changes and must be considered carefully even if it is a pure dielectric and any other interactions, like phonons, are neglected using valid approximations [3].

We use a generalized system of optical Bloch equations derived for an emitter in an ensemble of motionless similar particles embedded in a dielectric medium, which is transparent for the incident light [3-4]. The system is written with the effective rates of individual and collective radiative relaxation rates of the emitters, the Rabi frequency, and the effective transition-frequency shift caused by the presence of the dielectric host. The system's parameters are functions of the real and imaginary parts of the host's permittivity. The steady state solutions give hysteresis loops for the excited-state population of a two-level emitter if written as functions of the cw-laser field strength as shown in Fig. 1a. This population determines the total intensity of fluorescence and demonstrates the possibilities for switching between dim and bright fluorescence modes. If tuned near the hysteresis threshold points the system may be perturbed by either a fluctuation of the laser field or a small change in the density of the emitters. This perturbation is then likely to trigger the

*Corresponding author: lozing@phystech.edu 
switching. The transient regimes were simulated by solving the equations numerically. It appeared to be a long-time switching process and it was shown to be orders of magnitude ( $10^{4}$ and may be longer) longer than the excitation lifetime (see Fig. 1. b and c). When switching between the dim and the bright fluorescence occurs some transformations of the fluorescence spectrum are obviously expected. It was shown to be so by calculations of the fluorescence spectra both analytically and numerically. We assume, that the discussed effects could be observed experimentally by photon echo technique [5-7] or various methods of laser site-selective spectroscopy at low temperatures [8].

It appeared to be a long-time switching process and it was shown to be orders of magnitude $\left(10^{4}\right.$ and may be longer) longer than the excitation lifetime (Fig. 1. b and c).

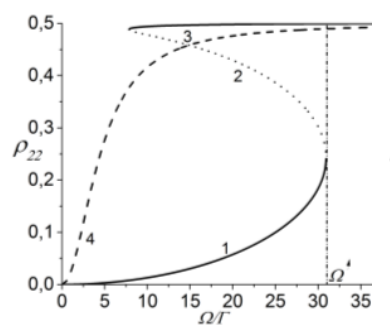

a)

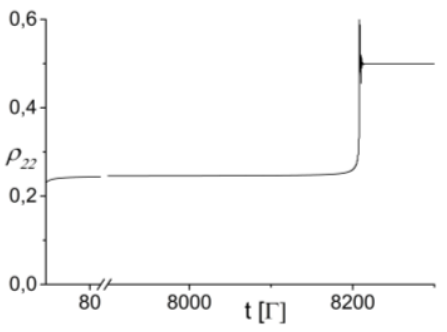

b)

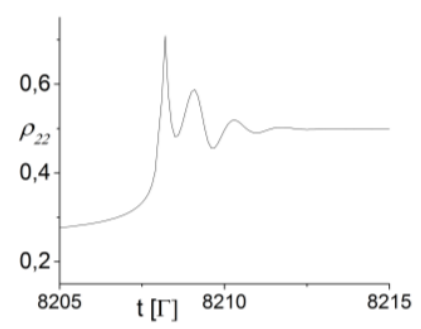

c)

Fig. 1. a) Steady state population of excited state, b) time-dependence of population after perturbing, c) time-dependence of population after perturbing (increased scale) near the transition point.

This work was supported by RFBR (proj. no. 16-02-01174).

\section{References}

1. L. Mandel, E. Wolf, Optical Coherence and Quantum Optics (Cambridge University Press, New York, 1995)

2. M.G. Gladush, D.V. Kuznetsov, V.K. Roerich, Eur. Phys. J. D 64, 511 (2011)

3. D.V. Kuznetsov, V.K. Roerich, M.G. Gladush, J. Exp. Theor. Phys. 113, 647 (2011)

4. M.G. Gladush, EPJ Web of Conferences 103, 02004 (2015)

5. Y.G. Vainer, A.V. Naumov, M.A. Kol'chenko, R.I. Personov, Phys. Stat. Sol. B 241, 3480 (2004)

6. K.R. Karimullin, M.V. Knyazev, A.V. Naumov, Bull. RAS: Physics 78, 1254 (2014)

7. K.R. Karimullin et al., Meas. Sci. Technol. 24, 027002 (2013)

8. A.V. Naumov, Physics-Uspekhi 56, 605 (2013) 